\title{
Simulation of an adaptive artificial neural network for power system security enhancement including control action
}

\begin{abstract}
This paper presents a new method for enhancing power system security, including a remedial action, using an artificial neural network (ANN) technique. The deregulation of electricity markets is still an essential requirement of modern power systems, which require the operation of an independent system driven by economic considerations. Power flow and contingency analyses usually take a few seconds to suggest a control action. Such delay could result in issues that affect system security. This study aims to find a significant control action that alleviates the bus voltage violation of a power system and to develop an automatic data knowledge generation method for the adaptive ANN. The developed method is proved to be a steady-state security assessment tool for supplying possible control actions to mitigate an insecure situation resulting from credible contingency. The proposed algorithm is successfully tested on the IEEE 9-bus and 39-bus test systems. A comparison of the results of the proposed algorithm with those of other conventional methods reveals that an ANN can accurately and instantaneously provide the required amounts of generation re-dispatch and load shedding in megawatts.
\end{abstract}

Keyword: Steady-state security assessment; Artificial neural network; Back-propagation; Remedial action; Contingency analysis 